\title{
Robotic and Laparoscopic Reconstructive Surgery in Children and Adults
}

MICHAEL C OST

\begin{tabular}{ll}
\hline EXTENT P/H & 302 pages, Hardback \\
PRICE/ISBN & $£ 144.00 \quad 9781603279130$ \\
PUBLISHER & Humana (New York), 2011 \\
REVIEWER & Henrik Steinbrecher \\
STAR RATING & $\star \star \star \star \star$
\end{tabular}

This book aims to be concise, comprehensive and a reference for all robotic and laparoscopic procedures in paediatric and adult urology. It achieves this admirably, despite being about 300 pages long, by producing succinct, wellstructured chapters that include the basic background of the pathology and procedure, and descriptions of equipment and operative steps, emphasising the avoidance of complications and thoroughly discussing advantages and disadvantages of various approaches.

The editor has brought together international experts and leaders in their field as well as some newer authors with up-to-date, precise and accurate material. Chapter references are comprehensive as is the index. Individual chapters can be dipped into and read perfectly well in isolation.

Reading the book in its entirety, the structure of each chapter is not exactly uniform but this does not detract from the important information contained therein. A few chapters have somewhat small or dark digital images and the labelling again is non-uniform throughout the book. In at least one image the labelling is incorrect. A number of chapters include online video clips via a listed website, which adds reality to the chapter prose. Again they are not uniform in structure, with some having audio descriptive accompaniment and others not, some being in many short clips and some in their entirety.

However, I thoroughly enjoyed reading this book and have no doubt that it is extremely useful for practising and developing laparoscopists for the safe basics of a procedure and for further tips and tricks regarding variations. 